\title{
Preparation of Hollow Fibre Composite Reverse Osmosis Membrane
}

\author{
Fengjuan Li \\ Key Lab of Hollow Fibre Membrane Materials \& Membrane Processes \\ (Tianjin Polytechnic University) Ministry of Education \\ Tianjin 300160, China \\ E-mail: 1i_fengjuan@163.com \\ Wei Wang (Corresponding author) \\ Key Lab of Hollow Fibre Membrane Materials \& Membrane Processes \\ (Tianjin Polytechnic University) Ministry of Education \\ Tianjin 300160, China \\ E-mail: wangwei7126@126.com \\ Qiang Ren \\ Oilfield Chemical Co . \\ CNOOC Energy Technology \& Service Oilfield Technology Services Co. \\ Tianjin 300452, China
}

\begin{abstract}
A novel thin-film composite (TFC) reverse osmosis (RO) membrane was developed by the interfacial polymerization (IP) of trimesoyl chloride (TMC) and m-phenylene diamine (MPD) on the hollow fibre ultrafiltration polysulphone (PS) membrane. The performance of the TFC membrane was optimized by studying the preparation parameters, which included monomer TMC concentration, monomer MPD concentration and alcohol additive in aqueous solution. The reverse osmosis performance of the resulting membrane was evaluated through permeation experiment with $1000 \mathrm{ppm}$ $\mathrm{NaCl}$ at $0.7 \mathrm{MPa}$. The results showed that the optimum concentration of MPD and TMC were $0.5 \mathrm{wt} \%$ and $3 \mathrm{~g} / \mathrm{L}$, respectively. To improve flux, alcohol was added to the aqueous phase, when certain concentration of alcohol was added to the aqueous solution, the flux of the prepared membrane increased from 5.0 to $9.1 \mathrm{~L} /\left(\mathrm{m}^{2} \cdot \mathrm{h}\right)$ without loss in salt rejection. The properties and structures of skin layer of RO composite membranes were characterized by AFM. It was found that IP had occurred and the separation layer was formed, and the novel reverse osmosis membrane appears to comprise a thicker, smoother and less cross-linking film structure. Additionally, the TFC membrane exhibits good long-term stability.
\end{abstract}

Keywords: Reverse osmosis, Trimesoyl chloride, M-phenylene diamine, Hollow fibre, Acohol additive

\section{Introduction}

The concept of reverse osmosis (RO) treatment was started through the development of the asymmetric cellulose acetate membrane by Loeb and Sourirajan in the 1960s. The second breakthrough in this field was achieved by the development of thin-film-composite (TFC) membrane with high flux by the interfacial polymerization. In recent years, reverse osmosis desalination has been explored as a viable technology for desalination of brackish water for industrial and civil use (Kosutic, Dolar et al. 2007; Hrayshat 2008; Radjenovic, Petrovic et al. 2008). Reverse osmosis membranes are extensively used worldwide or the desalination, softening and purification of brackish, sea and other surface waters for potable, industrial and agricultural use. For the development of RO membranes, two different techniques have been used: the phase inversion method for asymmetric membranes, and the interfacial polymerization for thin-film-composite (TFC) membranes (Matsuura 2001). TFC membrane preparation technique is based on interfacial polymerization 
(polycondensation) reaction between two monomers dissolved in water and hydrocarbon solvent, respectively (Roh, Kim et al. 2002; Freger 2003; Li, Zhang et al. 2007). Various benzenediamines and poly(aminostyrene) were interfacially reacted with various acryl chlorides to produce the thin skin layer. The obtained membrane showed typical trade-off behavior between the salt rejection and water permeability. Membrane prepared from trimesoyl chloride (TMC) and m-phenylene diamine (MPD) showed higher performance than usual membranes. Since water and the hydrocarbon solvent are immiscible, polymerization reaction takes place at the water/hydrocarbon interface. More specifically, the polymerization takes place at the organic phase side of the interface and not the aqueous phase side because of the highly non-favorable partition coefficient for the acid chloride which limits the availability of the acid chloride in the aqueous phase (Freger 2005). The thin film active layer ranging from $10 \mathrm{~nm}$ in thickness to several micrometers is quickly formed at the interface and strongly attached to the substrate (Freger and Srebnik 2003; Song, Sun et al. 2005).

A new concept for the preparation of thin-film-composite (TFC) reverse osmosis (RO)membrane by inter facial polymerization on porous polysulfone (PS) support using novel additives is reported. In order to improve the RO membrane performance, such as salt rejection and water flux, acohol was added to the aqueous phase. In this paper, the concentration of organic and aqueous phases was optimized since this parameter governs the amine monomer solubility and diffusivity in the reaction zone, which in fact affect the performance and morphology of the RO membrane (Ghosh, Jeong et al. 2008). We attempt to correlate MPD-TMC reaction to RO membrane separation performance (water flux, salt rejection) and interfacial characteristics (hydrophilicity, roughness). We also evaluate the addition of acohol to the aqueous MPD solution. These experiments allow us to satisfy our primary objective, that is, to elucidate the various interrelationships among reaction conditions and membrane properties. The properties and structures of skin layer of RO composite membranes were characterized by AFM. Finally, the impacts of operating pressure and the salt concentration on the properties of the RO membranes was discussed.

\section{Experimental}

\subsection{Chemicals and regents}

All chemicals used in this work are listed in Table 1. The chemicals, trimesoyl chloride (TMC), m-phenylenediamine (MPD), were used without further purification. Commercial UF polysulfone (PS) membranes of molecular weight cut-off (MWCO) 20,000 kD used as the support membrane were obtained from Tianjin Motimo membrane technology LTD. Monomer MPD (purity >99.5\%) was purchased from Tianjin Guanfu Fine Chemical Research Institute, China, and key functional monomer TMC (purity $>99.0 \%$ ) was obtained from Beijin Odyssey chemical Co. Ltd. China. Sodium chloride were provided by Tianjin Kermel Chemical Reagent Co. Ltd. China. All the other regents were of analytical grade unless specified.

\subsection{Membrane preparation}

A layer of an aqueous solution was formed on a microporous polysulphone supporting film by making contact for $5 \sim 10$ min of the solution with the supporting film and removing extra solution. The aqueous solution contained $0.5 \mathrm{wt} \% \mathrm{MPD}$. Then, a composite membrane was obtained by making contact with an organic solution containing $0.5 \mathrm{wt} \% \mathrm{TMC}$ for $30 \mathrm{~s}$ with the surface area of supporting film that was previously coated with the aqueous solution, and drying the film in a ventilation hood until heptane evaporated so that a skin layer was formed on the supporting film. The composite RO membrane was washed in deionized water and kept in glycerine overnight.

\subsection{Membrane performance testing}

Performance of the composite RO membranes was measured for all membrane samples using $1000 \mathrm{ppm} \mathrm{NaCl}$ at 0.7 $\mathrm{MPa}, 25^{\circ} \mathrm{C}$, respectively. The water flux was determined by direct measurement of the permeate flow. The water flux is given by

$$
\mathrm{F}=\mathrm{S} /(\mathrm{V} \times \mathrm{t})
$$

where $\mathrm{F}\left(\mathrm{L} / \mathrm{m}^{2} \cdot \mathrm{h}\right)$ is the water flux, $\mathrm{S}\left(\mathrm{m}^{2}\right)$ the effective area of membrane, $\mathrm{V}\left(\mathrm{m}^{3}\right)$ is the volume of permeate solution, $\mathrm{t}$ (h) the time.

The salt rejection rate was measured by the salt concentration in the permeate obtained through measurements of electrical conductance of the permeate and the feed using a conductance meter (DDS-11A, Shanghai Leici Instruments, China). The salt rejection of the membrane is defined as the following ratio

$$
\mathrm{R}(\%)=\left(1-\mathrm{C}_{\mathrm{p}} / \mathrm{C}_{0}\right) \times 100
$$

where $R$ is the salt rejection, $C_{p}(m g / L)$ the permeate concentration and $C_{0}(m g / L)$ the feed concentration.

The water flux and salt rejection of membranes were measured by collection the permeate for $30 \mathrm{~min}$ after stabilization for 30min. All membrane samples were prepared and tested in at least duplicated with a total of three membranes tests for RO performance, results of which have been averaged. Schematic drawing of the membrane equipment is illustrated 
in Figure.1.

\subsection{Membrane surface morphology}

The surface morphologies of composite membranes and substrate were observed with AFM. To prepare samples for AFM, the membranes were dried in vacuum at room temperature. The membranes were taken out of water and air-dried and they were ready for AFM observation.

\section{Results and discussion}

\subsection{The effect of MPD concentration}

In this research, reaction conditions, especially the concentration of monomers and relative reaction time, were optimized to obtain the most suitable one for the formation of the active top layer of the composite membrane. The effects of the concentration of MPD in aqueous on the performance of composite membrane was investigated at $25^{\circ} \mathrm{C}$, $0.3 \mathrm{MPa}$, with $1000 \mathrm{ppm} \mathrm{NaCl}$ aqueous solution as test solution. The results were shown in Figure.2. Figure.2. shows that the permeate flux declined and the rejection increased as the concentration of MPD in aqueous phase enhanced. But the declination of flux slowed down and the rejection became constant as the concentration of MPD over $0.5 \mathrm{wt} \%$.

\subsection{The effect of TMC concentration}

The relationship between the permeation properties of the membrane and the acyl chloride content was also investigated, and the result was illustrated in Figure. 3. Increasing the amount of acyl chloride in the polymerization solution results in a rapid increase in $\mathrm{NaCl}$ separation, correspondingly, the water flux decreases approximately exponentially as the amount of cross-linking agent is increased. The variation of composite membrane performance with the concentration of TMC in organic phase exhibited the same trend, but leveled off as the concentration of TMC exceeded $3 \mathrm{~g} / \mathrm{L}$, as shown in Fig. 3.

\subsection{The effect of acohol additive}

Acohol could be employed as an additive to control the roughness of the active layer, and then adjust the properties of the resulting composite RO membrane. Table 1 illustrates the performance of the resulting composite RO membranes to $1000 \mathrm{ppm} \mathrm{NaCl}$ solution. The permeate flux increased by $40 \%$ and the rejection wasn't decreased while the acohol was added into aqueous solution.

\subsection{Effect of operation pressure}

The effect of $\mathrm{NaC} 1$ concentration on $\mathrm{RO}$ rejection and flux are also considered. Experiments were carried out to study the effect of operating pressure, ranging between $0.4 \mathrm{MPa}$ and $0.7 \mathrm{MPa}$. The effect of operating pressure on RO rejection and flux are also considered was reported in Figure. 4. It shows that the flux of the RO membrane increase with an increase of operating pressure; the relationship between the flux and the operating pressure is nearly linear. It also shows that the rejection increases with increase in operating pressure for all the membranes, because ion transport due to convection becomes significant compared to diffusion. A high diffusive transport of salts through the membrane compared to convective transport may be the reason increase the rejection (Vaidya, Simaria et al. 2001).

\subsection{Effect of feed concentration on membrane performance}

The effect of $\mathrm{NaCl}$ concentration on $\mathrm{RO}$ rejection and flux are also considered. The feed concentration effect experiment was performed with the $\mathrm{RO}$ membrane by changing the $\mathrm{NaCl}$ concentration in the feed aqueous solution and the results of salt rejection and water flux to $\mathrm{NaCl}$ aqueous solution of different concentrations is shown in Figure.5. It shows that, as the feed $\mathrm{NaCl}$ concentration increases from 0.003 to $0.017 \mathrm{~mol} / \mathrm{L}$, the salt rejection of the membrane decreases slightly, while the flux of the membrane declines sharply because of the increase of osmotic pressure. It is also worth mentioning that the salt rejection of the membrane still remains more than $90 \%$ even the feed $\mathrm{NaCl}$ concentration is up to $0.01 \mathrm{~mol} / \mathrm{L}$.

\subsection{Membrane morphologies}

AFM images of the surface morphologies of RO membranes with a projection area of $10 \mu \mathrm{m} \times 10 \mu \mathrm{m}$ prepared from MPD and MPD-acohol were shown in Figure.6., in which the brightest point presented the highest point of the membrane and the dark regions presented the valleys. As shown in Fig.6., the order of the membrane roughness was MPD-acohol > MPD, and there was an obvious correlation of the water permeability with the surface roughness and surface area of the TFC membranes. It is obvious that the flux of the membrane increased in proportion to the increasing surface roughness and the enlarging surface area. The reason for this phenomenon might be that the rougher and larger surface area of the membranes made it possible to have contact with more water molecules in the given projected area, which attributed the higher flux.

\section{Conclusions}

A new concept for the preparation of TFC RO membrane by interfacial polymerization on hollow fibre ultrafiltration PS 
support is presented. The properties of the developed composite RO membranes could be adjusted by changing the concentration of the casting solution of the active layer, the concentration of the crosslinking agent, the addition of acohol, etc. The optimal polymerization conditions for the TFC RO membrane are as follows: MPD $=0.5 \mathrm{wt} \%$; $\mathrm{TMC}=3 \mathrm{~g} / \mathrm{L}$; added little acohol to aqueous solution. The permeate flux and the rejection increases while the flux increases as the operating pressure is increased in $\mathrm{NaCl}$ aqueous solution. However, when increased the concentration of $\mathrm{NaCl}$ aqueous solution, the salt rejection of the membrane decreases slightly, while the flux of the membrane declines sharply. Finally we found that added acohol to aqueous solution could improve the surface properties and of the TFC RO membrance, and increased the permeate flux considerably.

\section{References}

Freger, V. (2003). "Nanoscale heterogeneity of polyamide membranes formed by interfacial polymerization." Langmuir 19(11): 4791-4797.

Freger, V. (2005). "Kinetics of film formation by interfacial polycondensation." Langmuir 21(5): 1884-1894.

Freger, V. and S. Srebnik (2003). "Mathematical model of charge and density distributions in interfacial polymerization of thin films." Journal of Applied Polymer Science 88(5): 1162-1169.

Ghosh, A. K., B.-H. Jeong, et al. (2008). "Impacts of reaction and curing conditions on polyamide composite reverse osmosis membrane properties." Journal of Membrane Science 311(1-2): 34-45.

Hrayshat, E. S. (2008). "Brackish water desalination by a stand alone reverse osmosis desalination unit powered by photovoltaic solar energy." Renewable Energy 33(8): 1784-1790.

Kosutic, K., D. Dolar, et al. (2007). "Removal of antibiotics from a model wastewater by RO/NF membranes." Separation and Purification Technology 53(3): 244-249.

Li, L., S. Zhang, et al. (2007). "Polyamide thin film composite membranes prepared from 3,4',5-biphenyl triacyl chloride, 3,3',5,5'-biphenyl tetraacyl chloride and m-phenylenediamine." Journal of Membrane Science 289(1-2): 258-267.

Matsuura, T. (2001). "Progress in membrane science and technology for seawater desalination - a review." Desalination 134(1-3): 47-54.

Radjenovic, J., M. Petrovic, et al. (2008). "Rejection of pharmaceuticals in nanofiltration and reverse osmosis membrane drinking water treatment." Water Research 42(14): 3601-3610.

Roh, I. J., J. J. Kim, et al. (2002). "Mechanical properties and reverse osmosis performance of interfacially polymerized polyamide thin films." Journal of Membrane Science 197(1-2): 199-210.

Song, Y. J., P. Sun, et al. (2005). "Mechanisms of structure and performance controlled thin film composite membrane formation via interfacial polymerization process." Journal of Membrane Science 251(1-2): 67-79.

Vaidya, S. Y., A. V. Simaria, et al. (2001). "Reverse osmosis transport models evaluation: A new approach." Indian Journal of Chemical Technology 8(5): 335-343.

Table 1. The effect of acohol additive to the RO membrane salt rejection and water flux

\begin{tabular}{|l|l|l|}
\hline & \multicolumn{1}{|c|}{$\mathrm{F}\left(\mathrm{L} / \mathrm{m}^{2} \cdot \mathrm{h}\right)$} & \multicolumn{1}{c|}{$\mathrm{R}(\%)$} \\
\hline MPD & 5.0 & 93.9 \\
\hline Acohol additive & 9.1 & 97.8 \\
\hline
\end{tabular}




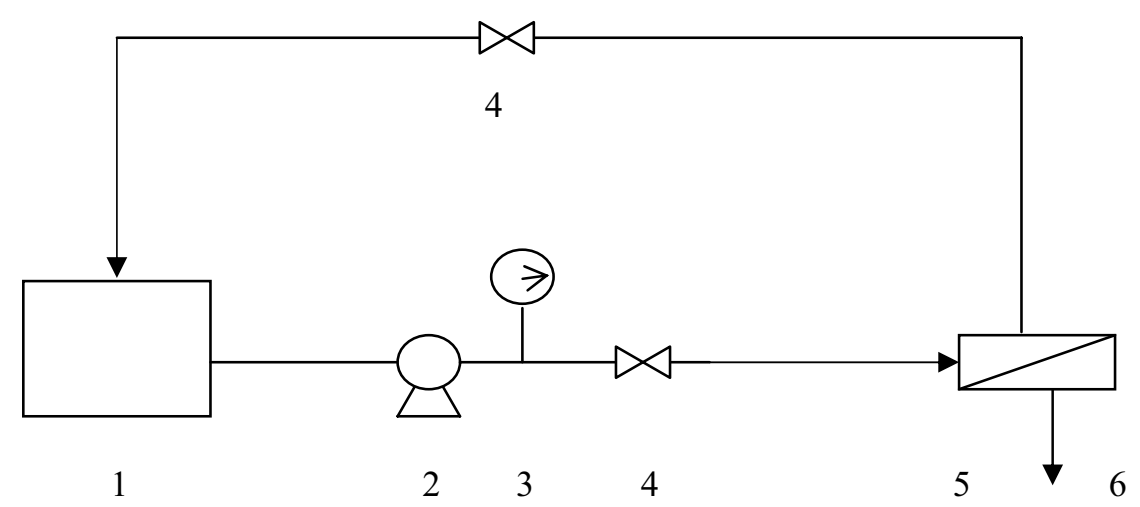

1 feed tank 2 diaphragm pump 3 pressure gauge 4 valve 5 RO membrane 6 permeate water Figure 1. Schematic drawing of the membrane equipment

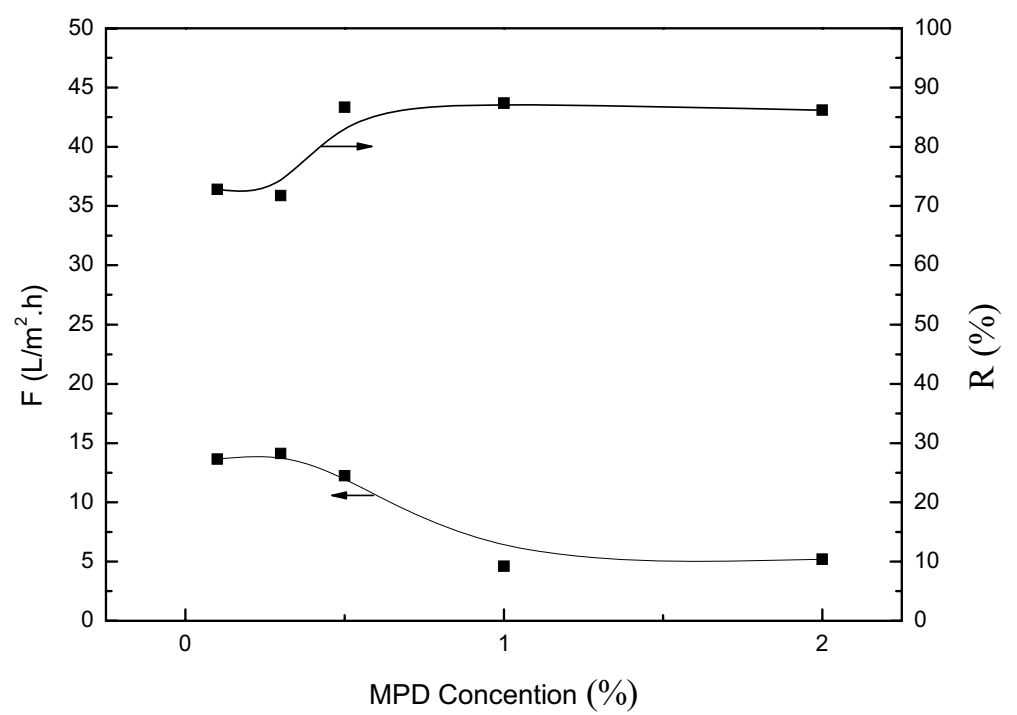

Figure 2. Effect of MPD concentration on salt rejection and water flux of the resulting membrane testing at $0.7 \mathrm{MPa}, 25^{\circ} \mathrm{C}$, with $1000 \mathrm{ppm} \mathrm{NaCl}$ aqueous solution $(\mathrm{TMC}=3 \mathrm{~g} / \mathrm{L})$ 


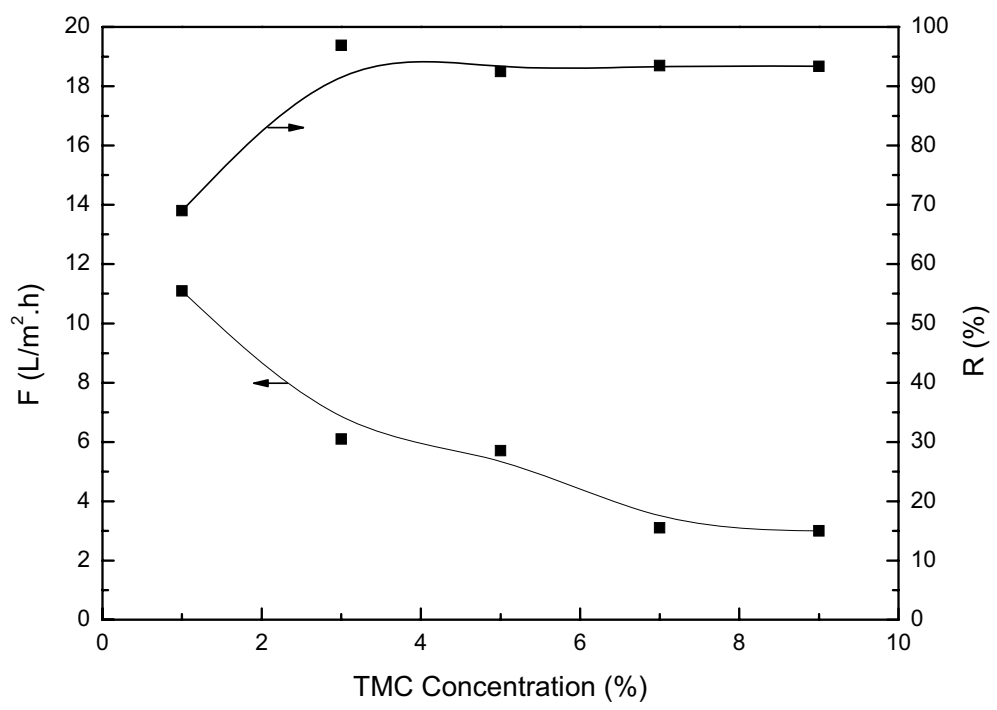

Figure 3. Effect on TMC concentration on salt rejection and water flux of the resulting membrane testing at $0.7 \mathrm{MPa}$, $25^{\circ} \mathrm{C}$, with $1000 \mathrm{ppm} \mathrm{NaCl}$ aqueous solution (MPD $=0.5 \mathrm{wt} \%$ )

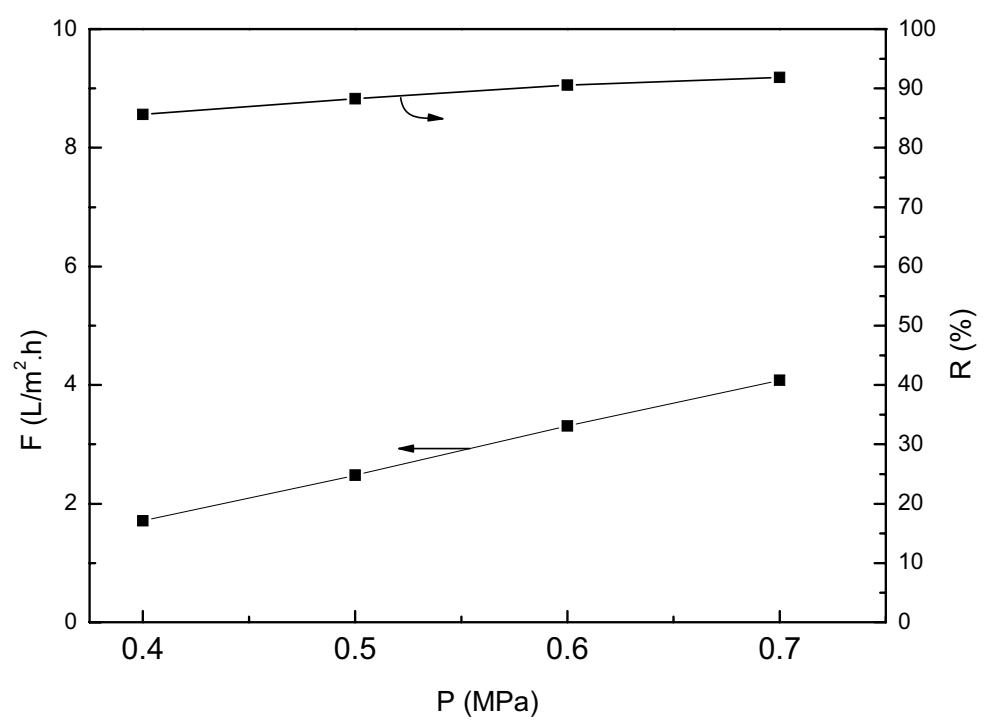

Figure 4. Effect of operation pressure on $\mathrm{RO}$ rejection and flux testing with $\mathrm{NaCl}$ aqueous solution at $0.7 \mathrm{MPa}, 25^{\circ} \mathrm{C}$ 


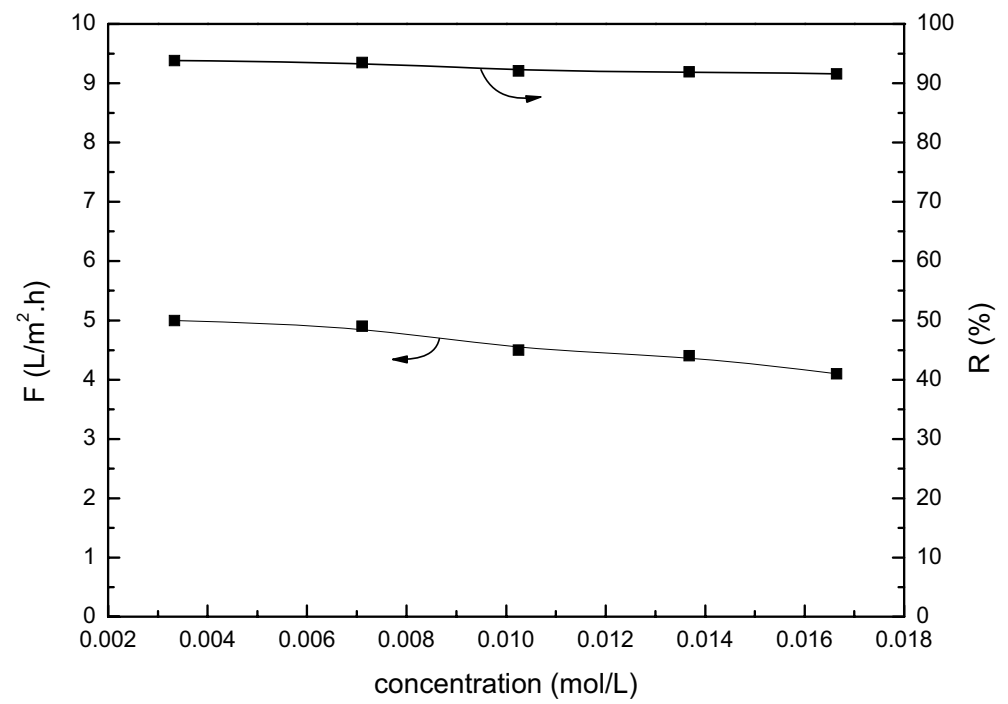

Figure 5. Effect of feed concentration on salt rejection and water flux testing with $\mathrm{NaCl}$ aqueous solution at $0.7 \mathrm{MPa}$, $25^{\circ} \mathrm{C}$

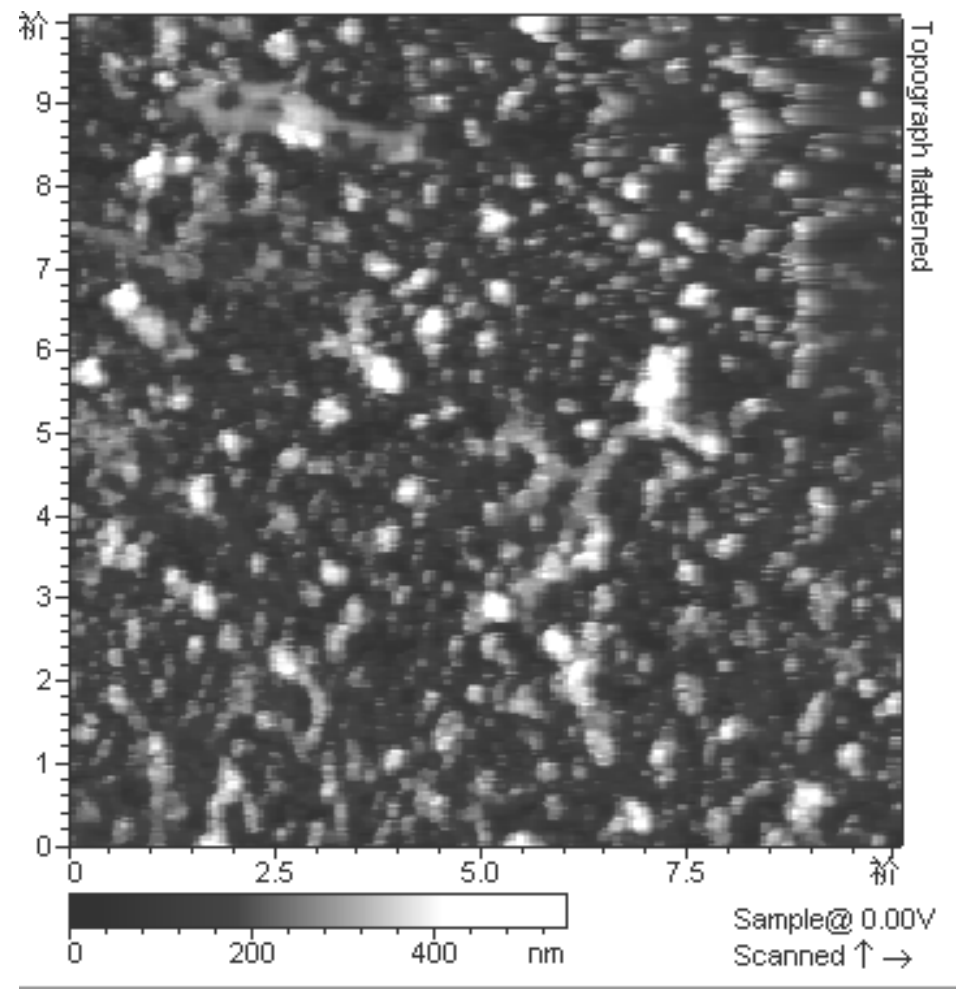

(a) 


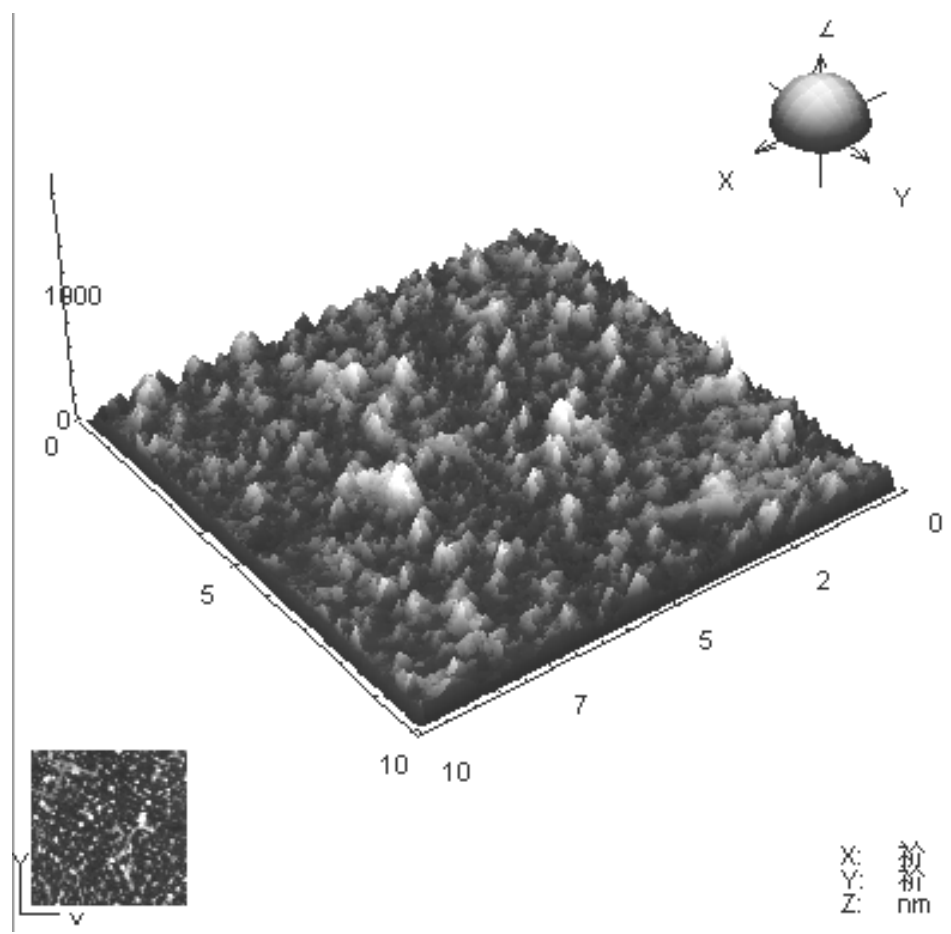

(b)

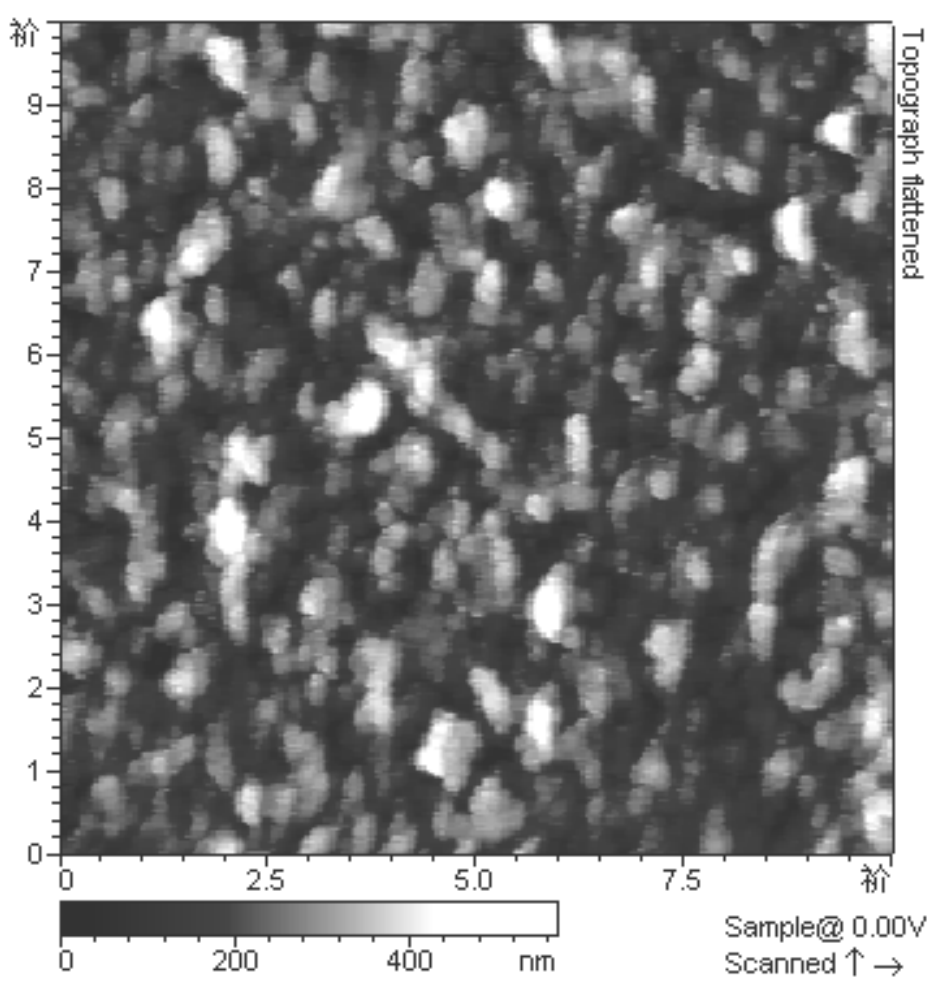

(c) 


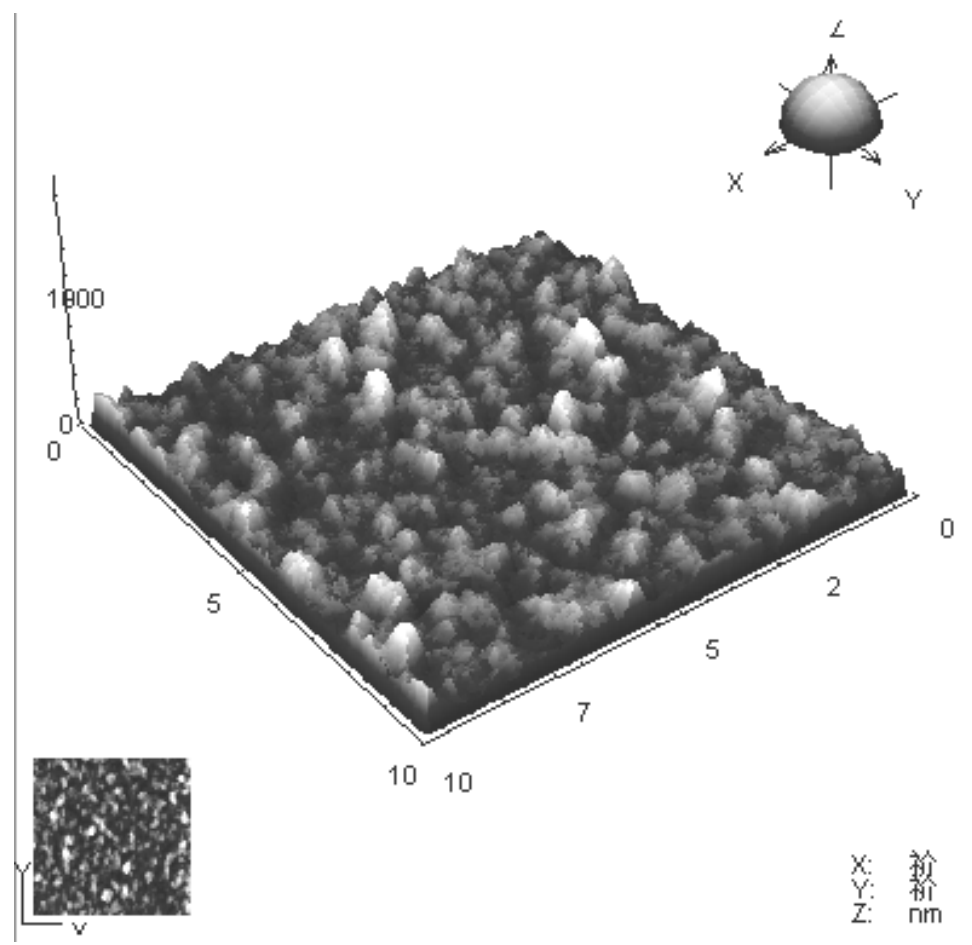

(d)

Figure 6. AFM images: (a) (b) the images of membranes prepared from MPD; (c) (d) the images of membranes prepared from MPD with acohol additive 